\title{
The Challenge of Diagnosing Heparin-Induced Thrombocytopenia with Negative Immunologic and Functional Assays
}

\author{
Glosser LD ${ }^{1}$, Knauss HM${ }^{2}$, J odeh $\mathbf{W}^{2}$ and Craig $\mathbf{D}^{2}$ \\ ${ }^{1}$ Department of Medical Education, University of Toledo \\ College of Medicine and Life Sciences, USA \\ ${ }^{2}$ Department of Internal Medicine, Saint J oseph Mercy \\ Ann Arbor Hospital, USA \\ *Corresponding author: Knauss HM, Department \\ of Medical Education, University of Toledo College of \\ Medicine and Life Sciences, 3000 Arlington Ave. Toledo \\ $\mathrm{OH} 43614$, USA
}

Received: April 25, 2021; Accepted: June 23, 2021; Published: June 30, 2021

\begin{abstract}
Heparin-Induced Thrombocytopenia (HIT) is a prothrombotic and potentially fatal immune complication of heparin therapy. HIT is challenging to diagnose, particularly in critically ill patients where multiple causes of thrombocytopenia must be considered. Diagnostic algorithms for HIT begin with a clinical assessment, followed by laboratory testing when indicated. If Platelet Factor-4 (PF4)/heparin immunoassay and Serotonin Release Assays (SRA) are negative, HIT is deemed unlikely and heparin therapy may be resumed. Current recommendations have excluded the next step in work up for thrombocytopenia after immunoassay and functional assays result negative despite worsening thrombocytopenia following heparin re-initiation. We present the case of an 85-year-old male with multiple comorbidities, found to have a clinical course consistent with HIT despite negative serologic and functional assay results. Our case highlights the challenge in diagnosing heparin-induced thrombocytopenia in a medically complex patient and demonstrates the need for standardized recommendations following negative laboratory results despite high clinical suspicion.
\end{abstract}

Keywords: Heparin-induced thrombocytopenia; Platelet Factor 4; Serotonin Release Assay; Immunoassay

\section{Abbreviations}

HIT: Heparin-Induced Thrombocytopenia; PF4: Platelet Factor 4; SRA: Serotonin Release Assay; LMWH: Low Molecular Weight Heparin; UFH: Unfractionated Heparin

\section{Introduction}

Heparin-Induced Thrombocytopenia (HIT) is a prothrombotic immune complication that occurs in up to $5 \%$ of patients treated with heparin therapy [1]. HIT arises when IgG antibodies target complexes of Platelet Factor 4 (PF4) and heparin, which subsequently induce widespread endothelial and platelet dysfunction [1]. HIT should be suspected in patients with declining platelet counts within 5-14 days of initiating Unfractionated (UFH) or Low-Molecular Weight Heparin (LMWH) [2]. When HIT is suspected, a clinical assessment such as the 4Ts scoring system is performed to determine the pretest probability [3]. If the pretest probability is intermediate or high, patients are switched to a non-heparin anticoagulant and an immunoassay is obtained [4]. Immunoassays are sensitive for detection of HIT and thus a negative result warrants heparin resumption [4]. However, false-negative results have been reported, especially early in the disease course. There is no standardized workup in patients with negative immunologic and functional assays in the context of high clinical suspicion. We present a patient with a clinical picture of heparin-induced thrombocytopenia despite negative serological results, highlighting the need to expand on current diagnostic guidelines.

\section{Case Presentation}

An 85 year-old male with a past medical history significant for transcatheter aortic valve replacement, congestive heart failure (CHF), chronic kidney disease, and two prior episodes of deep venous thrombosis presented to our hospital with a four day history of worsening lower extremity edema. He was admitted for CHF exacerbation complicated by cardiorenal syndrome and managed with bumetanide diuresis. Upon admission, his anticoagulation regimen was switched from his home dose of Apixaban (2.5mg twice daily) to a therapeutic heparin drip. His platelet count was 168 .

After 2 days of heparin therapy, his platelet count declined to 148. On day 6 of hospitalization, he was started on bridge to warfarin. On day 9 his BUN peaked at $146 \mathrm{mg} / \mathrm{dl}$, thus a peripherally inserted central catheter was placed for hemodialysis. His hospital course was further complicated by an E. coli urinary tract infection confirmed on day 12 and treated with IV ceftriaxone. On day 12, his platelet count also dropped to 84 from 118 the day prior. His 4Ts score was calculated to be 4 ( +1 platelet count fall by $30-50 \%$, +2 clear onset between 5-10 days after heparin, +1 for other possible causes of thrombocytopenia) indicating a moderate pretest probability for HIT at 14\% [3]. Following the HIT 4Ts score guidelines, heparin was discontinued and switched to argatroban. PF4/heparin antibody and serotonin release assays were ordered.

PF4/heparin antibody results returned negative on day 15 , measuring 0.37 (ref range $<0.4$ optical density). With a platelet count of 85 , anticoagulation was switched back to a heparin drip with 


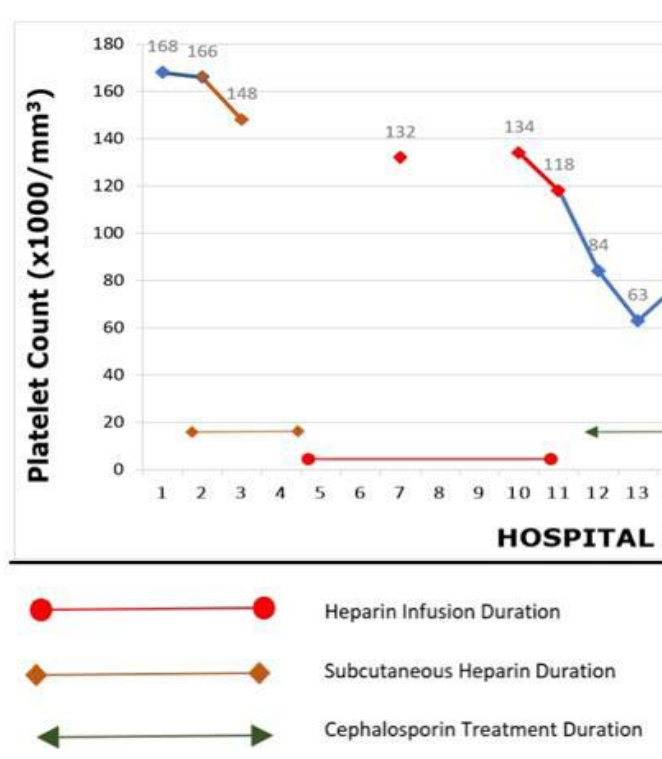

Figure 1: The Platelet Count.

bridge to warfarin, as argatroban was not preferred in the setting of acute kidney injury. Due to the potential of ceftriaxone-mediated thrombocytopenia, cephalexin was given on Day 16 for the last antibiotic dose treatment of UTI. Two days following resumption of heparin, his platelet count dropped to 60 . During the next 5 days of heparin bridge to warfarin, the platelet count ranged from 60-81 (Figure 1). On day 22, the bridge was completed and heparin was discontinued. Over the next 5 days after heparin discontinuation, his platelet count rose to 117 and the patient was discharged. The serotonin release assay later returned negative. Alternative etiologies of his thrombocytopenia were explored, and further workup for HIV, Covid-19, and Hepatitis B/C were negative.

\section{Discussion}

Current diagnostic guidelines for heparin-induced thrombocytopenia recommend immunoassay in patients with a 4Ts score of 4 and above [4]. The Platelet Factor-4 (PF4)/heparin immunoassay is reported to have a sensitivity of $>99 \%$, with a specificity of $30-70 \%$ [5]. If immunoassay is positive, a functional assay such as the SRA is ordered for confirmation [4]. Due to interlaboratory variability and lack of standardization, sensitivity of the SRA is suboptimal at $62-100 \%$ with a specificity of $89-100 \%$ [5]. The SRA further requires specialized laboratory settings not widely available, and results can take up to 1-2 weeks [6].

Limited data is available to support further workup for HIT in the context of negative laboratory results. According to the American Society of Hematology 2018 guidelines, a negative immunoassay in patients with low or intermediate pretest probability excludes HIT and warrants resumption of heparin [4]. Follow-up with repeat immunoassay, a different immunoassay, or functional assay is recommended only in patients with high pretest probability [4]. If further follow-up remains negative, there are no further diagnostic recommendations available.

There are several factors that impact the reliability of testing for HIT including time to testing, transfusion-related dilution of serum, and technical limitations. False-negative PF4/heparin antibodies and SRA testing have been reported early in disease course, followed by positive results on repeat testing [7-12]. Another case report demonstrated negative enzyme immunoassay and SRA results following massive blood transfusion, believed to be caused by dilution of HIT antibodies and should be considered with fluid administration [11]. Chan et al analyzed the utility of repeat HIT immunoassay testing in patients with initially negative GTI-PF4 Enzyme-Linked Immunosorbent Assay (ELISA) [12]. The study found that $80 \%$ of patients with an initial high Optical Density (OD) reading of $0.268-0.399$ had a positive ELISA on repeat testing [12]. Our patients OD reading of 0.37; unfortunately, repeat ELISA testing was not performed.

Our patient had several risk factors for HIT including unfractionated heparin use, duration of treatment, therapeutic dosing, and increased age $[2,13]$. This patient's platelets rose from 63 to 85 after the heparin was initially stopped for 3 days but dropped to 60 following re-initiation of heparin. 4 days after completing the final heparin dose, the platelets rose to 128 . No other cause of thrombocytopenia was established. Given the timing of heparin administration and subsequent platelet decline, our patient's clinical picture was congruent with HIT. The negative PF4/heparin and SRA may have been due to low levels of antibodies early in the disease course or laboratory error. Repeat testing was not ordered, but should be considered when the clinical picture conflicts with laboratory results.

\section{Conclusion}

Diagnosis of heparin-induced thrombocytopenia poses many challenges. Diagnostic guidelines do not account for clinical variability, technical limitations, and lack of laboratory standardization. Our case represents the need to account for false negative results and delineate when repeat testing is recommended. By fulfilling this need, future patients may benefit from avoidance of unnecessary medical interventions and associated costs. 


\section{References}

1. Cuker A, Cines DB. How I treat heparin-induced thrombocytopenia. Blood. 2012; 119: 2209-2218.

2. Salter BS, Weiner MM, Trinh MA, Heller J, Evans AS, Adams DH, et al. Heparin-Induced Thrombocytopenia: A Comprehensive Clinical Review. J Am Coll Cardiol. 2016; 67: 2519-2532.

3. Vatanparast R, Lantz S, Ward K, Crilley PA, Styler M. Evaluation of a pretest scoring system (4Ts) for the diagnosis of heparin-induced thrombocytopenia in a university hospital setting. Postgrad Med. 2012; 124: 36-42.

4. Cuker A, Arepally GM, Chong BH, Cines DB, Greinacher A, Gruel Y, et al American Society of Hematology 2018 guidelines for management of venous thromboembolism: heparin-induced thrombocytopenia. Blood Adv. 2018; 2 3360-3392.

5. Lee GM, Arepally GM. Diagnosis and management of heparin-induced thrombocytopenia. Hematol Oncol Clin North Am. 2013; 27: 541-563.

6. Minet V, Dogne JM, Mullier F. Functional Assays in the Diagnosis of HeparinInduced Thrombocytopenia: A Review. Molecules. 2017; 22: 617.

7. Omer T, Mullaguri N, George P, Newey CR. False-Negative Platelet Factor 4 Antibodies and Serotonin Release Assay and the Utility of Repeat Testing in the Diagnosis of Heparin-Induced Thrombocytopenia and Thrombosis. Case Rep Hematol. 2019; 2019: 1585014.
8. Jones CG, Pechauer SM, Curtis BR, Bougie DW, Irani MS, Dhakal B, et al. A Platelet Factor 4-Dependent Platelet Activation Assay Facilitates Early Detection of Pathogenic Heparin-Induced Thrombocytopenia Antibodies. Chest. 2017; 152: e77-e80.

9. Patel R, Varga C. Heparin-induced thrombocytopenia in a cardiac surgery patient with early and persistent thrombocytopenia and initial negative immunological and functional assays. Thromb Res. 2018; 169: 93-95.

10. Warkentin TE, Sheppard JA, Chu FV, Kapoor A, Crowther MA, Gangji A. Plasma exchange to remove HIT antibodies: dissociation between enzymeimmunoassay and platelet activation test reactivities. Blood. 2015; 125: 195198

11. Senzel L, Coldren D. Negative Heparin-Induced Thrombocytopenia Test Result after Massive Transfusion: Believe It or Not? Am J Clin Pathol. 2016; 145: 717-719.

12. Chan M, Malynn E, Shaz B, Uhl L. Utility of consecutive repeat HIT ELISA testing for heparin-induced thrombocytopenia. Am J Hematol. 2008; 83: 212 217.

13. Karnes $\mathrm{JH}$. Pharmacogenetics to prevent heparin-induced thrombocytopenia: what do we know? Pharmacogenomics. 2018; 19: 1413-1422. 